\title{
Path analysis and phenotypic correlation among yield components of soybean using environmental stratification methods
}

\author{
Mauricio Ferrari ${ }^{1}$, Ivan Ricardo Carvalho ${ }^{1}$, Alan Junior de Pelegrin ${ }^{1}$, Maicon Nardino ${ }^{1}$, Vinícius Jardel \\ Szareski ${ }^{1}$, Tiago Olivoto ${ }^{2}$, Tiago Corazza da Rosa ${ }^{1}$, Diego Nicolau Follmann ${ }^{2}$, Camila Pegoraro ${ }^{1}$, Luciano \\ Carlos da Maia ${ }^{1}$ and Velci Queiróz de Souza ${ }^{3}$
}

\author{
${ }^{1}$ Federal University of Pelotas - UFPel, Capão do Leão, RS, Brasil \\ ${ }^{2}$ Federal University of Santa Maria - UFSM, Santa Maria, RS, Brasil \\ ${ }^{3}$ Federal University of Pampa - UNIPAMPA, Dom Pedrito, RS, Brasil, Bolsista de Produtividade em Pesquisa CNPq
}

\section{*Corresponding authors:carvalho.irc@gmail.com}

\section{Abstract}

The main aim of this study was to investigate the phenotypic correlation of yield component traits using several environmental stratifications methods. We also aimed to propose cause and effect of relationships for grain yield components in soybean genotypes under several environmental conditions. The tests were conducted in the agricultural year of 2013/2014 in four growing sites in Rio Grande do Sul, Brazil. The experimental arrangement was randomized blocks in factorial scheme (11 x 4), consisting eleven soybean genotypes in four environments with four repetitions each. All the growing environments Tapera-RS, DerrubadasRS and Frederico Westphalen-RS were classified as favorable for soybean cultivation. The traits such as total number of pods per plant, number of branches and number of pods with 2-3 grains showed significant linear correlations with grain yield in both methods of analysis. The path analysis was applied under favorable and unfavorable environments to accurately estimate the direct and indirect effect of traits on soybean grain yield. The mass of a thousand grains and plant height were highly associated with grain yield but mostly influenced by environmental effects. The total number of pods should be prioritized for selecting superior soybean genotypes due to its direct and indirect effects on grain yield. It has shown constant in all environmental conditions. The direct effects of number of branches and number of pods (with one grain) presented distinct effects on yield in favorable and unfavorable environments.

Keywords: direct and indirect effects, genotypes, environment, interaction, favorable and unfavorable environment, Glycine max L. Abbreviations: GY_plot grain yield; MTG_mass of a thousand grains; PH_plant height; FPIH_first pod insertion height; TNP_total number of pods per plant; RN_number of ramifications; NOGP_number of one grain pods; NTWOGP_number of two grains pods; NTGP_number of three grains pods; DF_Degree of freedom; CV_coefficient of variation.

\section{Introduction}

The yield of soybean (Glycine max (L.) Merrill.) is a complex character, depending on the genotype and the growing environment known as genotypes $x$ environments ( $\mathrm{x}$ E) interaction, especially when grown in more than one environments. The genotype $x$ environment interaction consists of the differential performance of genotypes due to changes imposed by environments. In the other words, the response of each genotype is specific and different from another due to environmental changes (Carvalho et al., 2002). Being a complex trait of low heritability influenced by others, the environmental effects are even clearer.

Grain yield components may influence productivity directly and indirectly; therefore, changes in a given trait have a direct function on yield or on another trait associated with it. The traits that strongly associated with soybean grain yield correspond are the number of pods per plant, number of grains per pod and the mass of one thousand grains (Carvalho et al., 2002).
In order to understand and identify the associations between traits, linear correlation can be used. Linear correlation coefficients based on the phenotype indicate the linear phenotypic association between two characters (Cruz et al., 2004).

The existence and identification of the degree of correlation between traits are of fundamental importance for breeders, because improving a specific trait may cause simultaneous changes in another trait. It allows the use of indirect selection, if traits show high heritability (Falconer, 1981). The most common way to express linear correlations between two characters is the Pearson's linear correlation coefficient, which consists of the ratio of the two-variable joint variance known as covariance and the product of their respective standard deviations (Cruz et al., 2012).

The interpretation of a linear correlation magnitude and meaning is of great importance; however, it can result in misunderstandings in the selection strategy due to the possibility of a third trait or a group of traits present an 
indirect effect on this dependent trait (Carvalho et al., 2002; Cruz et al., 2012). Thus, Wright (1921) proposed the path analysis, which consists in the study of direct and indirect effects of traits on a dependent trait, obtaining these estimates through multiple regression equations. Thus, it is possible to deploy linear correlations into cause and effect estimates, and to apply indirect selection in less complex characters with greater heritability and easy measurement, leading to greater genetic progress, compared to the use of direct selection (Ramalho et al., 2012). Path analysis is widely used in soybean, in studies conducted by Shukla et al. (1998), Carvalho et al. (2002); Nogueira et al. (2012); Perini et al. (2012), Carvalho et al. (2015) and Szareski et al. (2015). The growing environment has great effect on phenotype expression; therefore, environmental characteristics can be the causes of correlation, once two traits are differently influenced by environmental conditions. Thus, environmental characteristics affect traits through different stimuli of physiological mechanisms (Falconer, 1981). However, in environments of similar conditions, the tendency is that correlations present similar direction and magnitude. In order to identify the existence of similar environmental patterns, some stratification analyzes can be used (Mendonça et al., 2007). Using the stratification, it is possible to minimize the effects of genotype $x$ environment interactions, because within each group of similar environments, the performance of the genotypes is constant and the interaction becomes non-significant (Ramalho et al., 1993).

The environmental stratification method proposed by Carneiro (1998) and implemented by Annicchiarico (1992), consists of the decomposition of the environmental index for favorable and unfavorable environments, being the environmental index defined as the difference between the average of the genotypes in each environment and the general average. Thus, indexes greater than or equal to zero indicate favorable environments, and negative indexes reveal unfavorable environments (Schmildt and Cruz, 2005). The analysis of factors is a model based on the multivariate technique and has the principle of formation of a matrix of factorial loads from the rotation of the factors (Carvalho et al., 2012). The application of this method results in the formation of sub-groups of environments based on the environmental indexes (lj). Then, the environments are classified as favorable, when they present positive values and unfavorable when the value is negative (Murakami and Cruz, 2004). In addition, positive factor loads greater than or equal to 0.7 indicate environments with high correlations within each factor, being possible to establish groups. Loads with magnitudes between 0.5 and 0.7 do not accurately define the formation of groups of environments, and magnitudes below 0.5 indicate the impossibility of forming groups of similar environments (Johnson and Wichern, 1992).

Genotype $x$ environment interaction influences the direct and indirect effects of traits associated with yield (Carvalho et al., 2002; Nogueira et al., 2012). Thereby, certain effects may be inflated when the phenotypes are jointly analyzed. In contrast, other effects can be masked because of the environmental characteristics. Thus, the most precise estimates are derived from the individual analyzes of each environment. However, this may increase the number of analyzes and the time required for interpreting the results.
As breeders seek the study of several environments simultaneously, they can classify them as favorable and unfavorable. In this sense, the stratification methods can both select and infer the relationships between traits considering more environments. The environmental stratification methods tend to group similar environments, where the genotype responses are more constant and they do not contribute interaction or reveal minimal effects. It would be possible to group these environments to deploy the correlations into estimates of direct and indirect yield component effects through path analysis.

Thus, the aim of this study was to investigate phenotypic correlations consistency using function of the environmental stratification method, and to propose cause and effect relationships based in the environmental stratification for grain yield components in soybean genotypes.

\section{Results and Discussion}

\section{Analysis of variance}

The joint variance analysis revealed significant interaction between genotypes and environments for the traits grain yield (GY), mass of a thousand grains (MTG), plant height $(\mathrm{PH})$, first pod insertion height (FPIH), number of ramifications (branches) (NR) number of one-grain pods (NOGP), number of two-grain pods (NTWOGP), and number of three-grain pods (NTGP) (Table 2). Therefore, the occurrence of interaction indicates that relative genotypes behavior was differently influenced by environmental conditions in each location (Silva and Duarte, 2006). Thus, the traits response is conditioned to each environment. In this case, extrapolation for all environments is not recommended. Therefore, it is necessary to determine the performance for each environment individually or jointly with all similar environments. According to Mendonça et al. (2007), the occurrence of significant genotype $x$ environment interaction justifies the environmental stratification to exploit the favorable environmental stimuli for the genotypes.

\section{Grouping of growing environments by the method of Annicchiarico (1992)}

One of the ways to minimize the genotype $x$ environments interaction is stratifying the environments to identify which environment is more similar according to phenotypic performance of genotypes (Ramalho et al., 2012). Environmental stratification of growing environments based on grain yield through the method of Annicchiarico (1992) classified the environments Tapera-RS, Derrubadas-RS and Frederico Westphalen-RS as favorable (Table 3), once they revealed positive environmental indexes and plot grain yield of average above the experiment general average (1211.79 g). The growing environment of Independência-RS presented negative environmental index and yield average inferior to the general average, being classified as unfavorable for cultivation of the soybean genotypes.

The result of factor analysis is presented on Table 4. The environments Tapera-RS, Derrubadas-RS and Frederico Westphalen-RS were grouped by factor 1 , because these environments presented factorial loads of $0.779 ; 0.920$ and 0.737 , respectively (Table 4 ). 
Table 1. Growing environments, geographic coordinates and edaphoclimatic characterization.

\begin{tabular}{|c|c|c|c|c|c|}
\hline Location & Latitude & Longitude & Altitude (m) & Soil $^{(1)}$ & Climate $^{(2)}$ \\
\hline Independência - RS & $27^{\circ} 51^{\prime} 18^{\prime \prime} \mathrm{S}$ & $54^{\circ} 17^{\prime} 13^{\prime \prime} \mathrm{O}$ & 315 & Dystrophic Red Latosol & $C f a$ \\
\hline Tapera - RS & $28^{\circ} 42^{\prime} 1^{\prime \prime} \mathrm{S}$ & $52^{\circ} 51^{\prime} 25^{\prime \prime} \mathrm{O}$ & 381 & Dystropherric Red Latosol & $C f a$ \\
\hline Derrubadas - RS & $27^{\circ} 16^{\prime} 63^{\prime \prime} \mathrm{S}$ & $53^{\circ} 47^{\prime} 33^{\prime \prime} 0$ & 430 & Eutropherric Red Nitosol & $c f a$ \\
\hline Frederico Westphalen - RS & $27^{\circ} 39^{\prime} 56^{\prime \prime} \mathrm{S}$ & $53^{\circ} 42^{\prime} 94^{\prime \prime} 0$ & 490 & Dystrophic Red Latosol & $c f a$ \\
\hline
\end{tabular}

${ }^{(1)}$ Classification according to methodology proposed by Santos et al. (2006); ${ }^{(2)}$ Climatic classification according to Maluf (2000).

Table 2. Summary of mean square of joint variance analysis, with deployment of genotype $x$ environment interaction for the traits plot grain yield (GY) $\left(\mathrm{kg} \mathrm{plot}^{-1}\right)$, mass of a thousand grains (MTG) $(\mathrm{g})$, plant height $(\mathrm{PH})(\mathrm{cm})$, first pod insertion height (FPIH) (cm), total number of pods per plant (TNP) number of ramifications (NR) (unit), number of one grain pods (NOGP) (unit), number of two grains pods (NTWOGP) (unit), and number of three grains pods (NTGP) (unit).

\begin{tabular}{|c|c|c|c|c|c|c|c|c|c|c|}
\hline \multirow{2}{*}{ Sources of variation } & \multirow{2}{*}{$\mathrm{DF}^{(1)}$} & \multicolumn{9}{|c|}{ Mean Square } \\
\hline & & $\overline{G Y}$ & MTG & $\mathrm{PH}$ & FPIH & TNP & NR & NOGP & NTWOGP & NTGP \\
\hline Bloc/environments & 12 & 624.3 & 46.3 & 131.8 & 16.1 & 38.1 & 0.2 & 1.6 & 5.891 & 16.8 \\
\hline Genotypes (G) & 10 & $6328.7^{*}$ & $4274.9^{*}$ & $1486.0^{*}$ & $180.2^{*}$ & $741.2^{*}$ & $12.6^{*}$ & $28.5^{*}$ & $303.1^{*}$ & $85.9 *$ \\
\hline Environment $(\mathrm{A})$ & 3 & $8956.2^{*}$ & $9696.4^{*}$ & $12386.7^{*}$ & $1069.9 *$ & $1574.8^{*}$ & $13.3^{*}$ & $40.2^{*}$ & $247.6^{*}$ & $314.4^{*}$ \\
\hline GxE & 30 & $651.6^{*}$ & $249.0^{*}$ & $47.6^{*}$ & $10.9 *$ & $31.9^{\text {ns }}$ & $0.8^{*}$ & $4.2^{*}$ & $15.6^{*}$ & $14.0^{*}$ \\
\hline Residue & 120 & 404.4 & 49.5 & 30.4 & 5.7 & 24.9 & 0.2 & 1.6 & 5.3 & 8.3 \\
\hline $\mathrm{CV}^{(2)}(\%)$ & - & 12.98 & 4.382 & 6.03 & 9.57 & 11.84 & 18.489 & 25.75 & 13.88 & 14.33 \\
\hline
\end{tabular}

* Significant to 0.05 of probability through test $F ;{ }^{(n s)}$ non-significant to 0.05 of probability through test $F ;{ }^{(1)} \mathrm{DF}$ : Degree of freedom; ${ }^{(2)} \mathrm{CV}$ : coefficient of variation.

Table 3. Grouping of growing environments by the method of Annicchiarico (1992), based on the trait plot grain yield $\left(\mathrm{kg} \mathrm{par}^{-1}\right)$, for 11 soybean genotypes cultivated in for environments in Rio Grande do Sul.

\begin{tabular}{lccc}
\hline Growingenvironments & Average $\left(\mathrm{g} \mathrm{plot}^{-1}\right)$ & Environmental index & Class \\
\hline Independência-RS & 1057.58 & -21.068 & Unfavorable \\
Tapera- RS & 1259.47 & 4.945 & Favorable \\
Derrubadas - RS & 1278.12 & 10.549 & Favorable \\
Frederico Westphalen -RS & 1252.00 & 5.574 & Favorable \\
\hline
\end{tabular}

Table 4. Environmental stratification based on the trait plot grain yield $\left(\mathrm{Kg} \mathrm{par}^{-1}\right)$ achieved through factor analysis for 11 soybean genotypes in four environments in Rio Grande do Sul.

\begin{tabular}{|c|c|c|c|c|c|c|c|}
\hline \multicolumn{3}{|c|}{ Eigenvalueestimates } & \multirow{2}{*}{ Growing environments } & \multicolumn{4}{|c|}{ Factorial loads after rotation } \\
\hline Root & (\%) Root & (\%) Accumulated & & Factor 1 & Factor 2 & Com* & $\mathrm{El} \mathrm{I}^{* *}$ \\
\hline 3.134 & 78.36 & 78.36 & Independência-RS & 0.269 & 0.938 & 0.95 & -21.067 \\
\hline 0.507 & 12.66 & 91.02 & Tapera - RS & 0.779 & 0.508 & 0.86 & 4.945 \\
\hline 0.29 & 7.26 & 98.28 & Derrubadas - RS & 0.92 & 0.194 & 0.88 & 10.548 \\
\hline 0.069 & 1.72 & 100 & Frederico Westphalen -RS & 0.737 & 0.6267 & 0.94 & 5.574 \\
\hline
\end{tabular}

${ }^{*}$ Communality; ${ }^{* *}$ Environmental index.

Table 5. Estimates of Pearson's linear correlation for joint analysis of environments (joint), for environmental stratification (Fav. Env. and Unf. Env.) for nine traits of soybean grain yield.

\begin{tabular}{|c|c|c|c|c|c|c|c|c|c|}
\hline & & $G Y^{(1)}$ & MTG & $\mathrm{PH}$ & $\mathrm{FPIH}$ & TNP & NR & NOGP & NTWOGP \\
\hline & joint $^{(2)}$ & $0.187^{*}$ & - & & & & & & \\
\hline \multirow[t]{3}{*}{ MTG } & Fav. Env. & $0.188^{*}$ & - & & & & & & \\
\hline & Unf. Env. & 0.180 & - & & & & & & \\
\hline & joint & $0.349 *$ & $0.316^{*}$ & - & & & & & \\
\hline \multirow[t]{3}{*}{$\mathrm{PH}$} & Fav. Env. & 0.118 & $0.460 *$ & - & & & & & \\
\hline & Unf. Env. & 0.196 & 0.076 & - & & & & & \\
\hline & joint & 0.139 & $0.542^{*}$ & $0.741^{*}$ & - & & & & \\
\hline \multirow[t]{3}{*}{ FPIH } & Fav. Env. & -0.077 & $0.623^{*}$ & $0.691^{*}$ & - & & & & \\
\hline & Unf. Env. & 0.157 & $0.548 *$ & $0.384 *$ & - & & & & \\
\hline & joint & $0.661 *$ & $-0.482 *$ & 0.109 & $-0.288^{*}$ & - & & & \\
\hline \multirow[t]{3}{*}{ TNP } & Fav. Env. & $0.625^{*}$ & $-0.510 *$ & $-0.207 *$ & $-0.546 *$ & - & & & \\
\hline & Unf. Env. & $0.576^{*}$ & $-0.574 *$ & 0.265 & $-0.308 *$ & - & & & \\
\hline & joint & $0.494 *$ & -0.082 & $-0.144^{*}$ & $-0.320^{*}$ & $0.583^{*}$ & - & & \\
\hline \multirow[t]{3}{*}{ NR } & Fav. Env. & $0.592^{*}$ & -0.112 & $-0.216^{*}$ & $-0.398 *$ & $0.665 *$ & - & & \\
\hline & Unf. Env. & $0.464 *$ & 0.011 & 0.148 & -0.023 & $0.531 *$ & - & & \\
\hline & joint & $0.342^{*}$ & $-0.426^{*}$ & 0.121 & $-0.208^{*}$ & $0.722^{*}$ & $0.415^{*}$ & - & \\
\hline \multirow[t]{3}{*}{ NOGP } & Fav. Env. & $0.277^{*}$ & $-0.417^{*}$ & -0.106 & $-0.414^{*}$ & $0.687^{*}$ & $0.438^{*}$ & - & \\
\hline & Unf. Env. & 0.222 & $-0.598 *$ & 0.096 & -0.222 & $0.744 *$ & $0.486 *$ & - & \\
\hline & joint & $0.454^{*}$ & $-0.512 *$ & 0.004 & $-0.385^{*}$ & $0.885 *$ & $0.588^{*}$ & $0.716^{*}$ & - \\
\hline \multirow[t]{3}{*}{ NTWOGP } & Fav. Env. & $0.441^{*}$ & $-0.483 *$ & $-0.187^{*}$ & $-0.563 *$ & $0.889 *$ & $0.633^{*}$ & $0.685^{*}$ & - \\
\hline & Unf. Env. & $0.368 *$ & $-0.673 *$ & 0.056 & $-0.401 *$ & $0.889 *$ & $0.526 *$ & $0.790 *$ & - \\
\hline & joint & $0.730^{*}$ & $-0.245^{*}$ & $0.149 *$ & -0.069 & $0.741 *$ & $0.403^{*}$ & $0.292 *$ & $0.406^{*}$ \\
\hline \multirow[t]{2}{*}{ NTGP } & Fav. Env. & $0.674 *$ & $-0.306 *$ & -0.147 & $-0.277^{*}$ & $0.708 *$ & $0.467 *$ & $0.208^{*}$ & $0.361^{*}$ \\
\hline & Unf. Env. & $0.803 *$ & -0.150 & $0.343^{*}$ & -0.046 & $0.743 *$ & $0.365^{*}$ & $0.320 *$ & $0.453^{*}$ \\
\hline
\end{tabular}

"Coefficients of Pearson's linearcorrelation significant to $5.00 \%$ of probability. ${ }^{(1)} \mathrm{GY}$ : plot grain yield; MTG: mass of a thousand grains; PH: plant height; FPIH: first pod insertion height; TNP: total number of pods per plant; RN: number of ramifications; NOGP: number of one grain pods; NTWOGP: number of two grains pods; NTGP: number of three grains pods. ${ }^{(2)}$ joint: Pearson's linear joint correlation ( $n=176$ ); Fav. Env.: Pearson's linear correlation for favorable environments ( $n=132)$; and Unf. Env.: Pearson's linear correlation for unfavorable environments ( $n=44)$. 
Table 6. Estimates of Pearson's linear correlation for growing environment (Independência-RS, Tapera-RS, Derrubadas-RS, Frederico Westphalen-RS) for nine components of soybean grain yield.

\begin{tabular}{|c|c|c|c|c|c|c|c|c|c|}
\hline & Environments & $G Y^{(1)}$ & MTG & $\mathrm{PH}$ & $\mathrm{FPIH}$ & TNP & NR & NOGP & NTWOGP \\
\hline \multirow{4}{*}{ MTG } & Independência-RS ${ }^{(2)}$ & 0.180 & - & & & & & & \\
\hline & Tapera-RS & $0.345^{*}$ & - & & & & & & \\
\hline & Derrubadas-RS & 0.256 & - & & & & & & \\
\hline & Frederico Westphalen-RS & $0.402 *$ & - & & & & & & \\
\hline \multirow{4}{*}{$\mathrm{PH}$} & Independência-RS & 0.196 & 0.076 & - & & & & & \\
\hline & Tapera-RS & 0.027 & 0.085 & - & & & & & \\
\hline & Derrubadas-RS & $0.474 *$ & 0.282 & - & & & & & \\
\hline & Frederico Westphalen-RS & 0.171 & 0.297 & - & & & & & \\
\hline \multirow{4}{*}{ FPIH } & Independência-RS & 0.157 & $0.548^{*}$ & $0.384^{*}$ & - & & & & \\
\hline & Tapera-RS-RS & -0.024 & $0.344^{*}$ & $0.573^{*}$ & - & & & & \\
\hline & Derrubadas-RS-RS & -0.083 & 0.234 & $0.321^{*}$ & - & & & & \\
\hline & Frederico Westphalen-RS & -0.003 & $0.577^{*}$ & $0.537^{*}$ & - & & & & \\
\hline \multirow{4}{*}{ TNP } & Independência-RS & $0.576^{*}$ & $-0.574^{*}$ & 0.265 & $-0.308^{*}$ & - & & & \\
\hline & Tapera-RS & $0.705^{*}$ & $-0.322 *$ & 0.024 & -0.286 & - & & & \\
\hline & Derrubadas-RS & $0.613^{*}$ & $-0.425^{*}$ & 0.162 & $-0.467^{*}$ & - & & & \\
\hline & Frederico Westphalen-RS & $0.760 *$ & -0.119 & 0.221 & $-0.332 *$ & - & & & \\
\hline \multirow{4}{*}{ NR } & Independência-RS & $0.464^{*}$ & 0.011 & 0.148 & -0.023 & $0.530 *$ & - & & \\
\hline & Tapera-RS & $0.736^{*}$ & 0.194 & -0.056 & -0.294 & $0.621^{*}$ & - & & \\
\hline & Derrubadas-RS & $0.598^{*}$ & -0.046 & 0.163 & -0.215 & $0.709 *$ & - & & \\
\hline & Frederico Westphalen-RS & $0.618^{*}$ & 0.240 & 0.293 & -0.001 & $0.524^{*}$ & - & & \\
\hline \multirow{4}{*}{ NOGP } & Independência-RS & 0.222 & $-0.598^{*}$ & 0.096 & -0.222 & $0.744^{*}$ & $0.486^{*}$ & - & \\
\hline & Tapera-RS & 0.284 & $-0.390 *$ & 0.108 & -0.243 & $0.623^{*}$ & $0.388^{*}$ & - & \\
\hline & Derrubadas-RS & 0.278 & $-0.480 *$ & 0.042 & $-0.406^{*}$ & $0.700 *$ & $0.457^{*}$ & - & \\
\hline & Frederico Westphalen-RS & 0.236 & -0.099 & 0.275 & -0.212 & $0.586^{*}$ & 0.197 & - & \\
\hline \multirow{4}{*}{ NTWOGP } & Independência-RS & $0.368^{*}$ & $-0.673^{*}$ & 0.056 & $-0.401^{*}$ & $0.889 *$ & $0.526^{*}$ & $0.790^{*}$ & - \\
\hline & Tapera-RS & $0.467^{*}$ & $-0.401^{*}$ & 0.074 & $-0.413^{*}$ & $0.850 *$ & $0.589 *$ & $0.639 *$ & - \\
\hline & Derrubadas-RS & $0.354^{*}$ & $-0.505^{*}$ & -0.024 & $-0.606^{*}$ & $0.888^{*}$ & $0.625^{*}$ & $0.667^{*}$ & - \\
\hline & Frederico Westphalen-RS & $0.580^{*}$ & -0.152 & 0.213 & $-0.357^{*}$ & $0.868^{*}$ & $0.534^{*}$ & $0.613^{*}$ & - \\
\hline \multirow{4}{*}{ NTGP } & Independência-RS & $0.803^{*}$ & -0.150 & $0.343^{*}$ & -0.046 & $0.743^{*}$ & $0.365^{*}$ & $0.320^{*}$ & $0.453^{*}$ \\
\hline & Tapera-RS & $0.727^{*}$ & 0.158 & -0.062 & 0.134 & $0.515^{*}$ & $0.369 *$ & -0.004 & 0.079 \\
\hline & Derrubadas-RS & $0.760 *$ & -0.052 & $0.402 *$ & -0.031 & $0.678^{*}$ & $0.521 *$ & 0.249 & $0.303^{*}$ \\
\hline & Frederico Westphalen-RS & $0.681^{*}$ & -0.018 & 0.083 & -0.095 & $0.642^{*}$ & $0.331^{*}$ & -0.086 & 0.261 \\
\hline
\end{tabular}

number of pods per plant: RN: number of ramifications; NOGP: number of one grain pods; NTWOGP: number of two grains pods; NTGP: number of three grains pods. ${ }^{(2)}$ Pearson's linear correlation by growing environment Independência, Tapera, Derrubadas e Frederico Westphalen ( $\mathrm{n}=44)$.

Table 7. Estimates of direct and indirect phenotypic effects for joint analysis of environments (joint), environmental stratification (FVEV and UFEV), and for environments Tapera-RS (TAP), Derrubadas-RS (DER), Frederico Westphalen-RS (F.WE) and Independência-RS (IND), in eleven soybean genotypes using plot grain yield $\left(\mathrm{kg} \mathrm{par}^{-1}\right.$ ) as dependent character.

\begin{tabular}{|c|c|c|c|c|c|c|c|}
\hline \multirow{2}{*}{ Effects } & \multirow{2}{*}{ joint } & \multirow{2}{*}{ FVEV } & \multicolumn{3}{|c|}{ FAV. ENV. } & \multirow{2}{*}{ UFEV } & \multirow{2}{*}{$\frac{\text { UNF. ENV. }}{\text { IND }}$} \\
\hline & & & $\operatorname{TAP}^{(2)}$ & DER & F.WE & & \\
\hline Direct of $\mathrm{MTG}^{(1)}$ & 0.575 & 0.619 & 0.481 & 0.504 & 0.468 & 0.576 & 0.576 \\
\hline Ind. through $\mathrm{PH}$ & 0.005 & 0.024 & -0.002 & 0.018 & -0.063 & -0.017 & -0.017 \\
\hline Ind.through FPIH & 0.025 & -0.017 & 0.004 & 0.019 & 0.054 & 0.100 & 0.100 \\
\hline Ind.through TNP & -0.149 & -0.200 & -0.102 & -0.113 & -0.073 & -0.220 & -0.220 \\
\hline Ind.through NR & 0.000 & -0.007 & 0.023 & -0.001 & 0.028 & -0.001 & -0.001 \\
\hline Ind.through NOGP & -0.018 & -0.012 & -0.032 & -0.051 & 0.007 & 0.038 & 0.038 \\
\hline Ind.through NTWOGP & -0.125 & -0.070 & -0.097 & -0.099 & -0.017 & -0.212 & -0.212 \\
\hline Ind.through NTGP & -0.129 & -0.151 & 0.066 & -0.025 & -0.005 & -0.088 & -0.088 \\
\hline Total & 0.187 & 0.189 & 0.345 & 0.256 & 0.402 & 0.181 & 0.181 \\
\hline Directof AP & 0.016 & 0.051 & -0.021 & 0.065 & -0.213 & -0.222 & -0.222 \\
\hline Ind.through MTG & 0.182 & 0.285 & 0.041 & 0.142 & 0.139 & 0.044 & 0.044 \\
\hline Ind.through FPIH & 0.034 & -0.018 & 0.006 & 0.026 & 0.051 & 0.070 & 0.070 \\
\hline Ind.through TNP & 0.034 & -0.081 & 0.008 & 0.043 & 0.135 & 0.101 & 0.101 \\
\hline Ind.through NR & -0.001 & -0.014 & -0.007 & 0.003 & 0.035 & -0.008 & -0.008 \\
\hline Ind.through NOGP & 0.005 & -0.003 & 0.009 & 0.004 & -0.018 & -0.006 & -0.006 \\
\hline Ind.through NTWOGP & 0.001 & -0.027 & 0.018 & -0.005 & 0.024 & 0.018 & 0.018 \\
\hline Ind.through NTGP & 0.079 & -0.073 & -0.026 & 0.196 & 0.021 & 0.201 & 0.201 \\
\hline Total & 0.350 & 0.119 & 0.028 & 0.474 & 0.171 & 0.196 & 0.196 \\
\hline Direct of FPIH & 0.045 & -0.027 & 0.011 & 0.081 & 0.094 & 0.182 & 0.182 \\
\hline Ind.through MTG & 0.312 & 0.386 & 0.166 & 0.118 & 0.270 & 0.316 & 0.316 \\
\hline Ind.through PH & 0.012 & 0.035 & -0.012 & 0.021 & -0.115 & -0.085 & -0.085 \\
\hline Ind.through TNP & -0.089 & -0.214 & -0.090 & -0.124 & -0.203 & -0.118 & -0.118 \\
\hline Ind.through NR & -0.002 & -0.026 & -0.035 & -0.003 & 0.000 & 0.001 & 0.001 \\
\hline Ind.through NOGP & -0.009 & -0.012 & -0.020 & -0.043 & 0.014 & 0.014 & 0.014 \\
\hline Ind.through NTWOGP & -0.094 & -0.082 & -0.100 & -0.119 & -0.041 & -0.126 & -0.126 \\
\hline
\end{tabular}




\begin{tabular}{|c|c|c|c|c|c|c|c|}
\hline Ind.through NTGP & -0.037 & -0.137 & 0.057 & -0.015 & -0.024 & -0.027 & -0.027 \\
\hline Total & 0.139 & -0.077 & -0.024 & -0.084 & -0.003 & 0.157 & 0.157 \\
\hline Direct of TNP & 0.309 & 0.392 & 0.315 & 0.266 & 0.609 & 0.383 & 0.383 \\
\hline Ind.through MTG & -0.278 & -0.316 & -0.155 & -0.215 & -0.056 & -0.331 & -0.331 \\
\hline Ind.through PH & 0.002 & -0.011 & -0.001 & 0.010 & -0.047 & -0.059 & -0.059 \\
\hline Ind.through FPIH & -0.013 & 0.015 & -0.003 & -0.038 & -0.031 & -0.056 & -0.056 \\
\hline Ind.through NR & 0.003 & 0.044 & 0.074 & 0.011 & 0.062 & -0.030 & -0.030 \\
\hline Ind.through NOGP & 0.030 & 0.020 & 0.050 & 0.074 & -0.039 & -0.048 & -0.048 \\
\hline Ind.through NTWOGP & 0.216 & 0.129 & 0.206 & 0.174 & 0.099 & 0.280 & 0.280 \\
\hline Ind.through NTGP & 0.391 & 0.350 & 0.216 & 0.330 & 0.160 & 0.434 & 0.434 \\
\hline Total & 0.662 & 0.626 & 0.705 & 0.614 & 0.761 & 0.576 & 0.576 \\
\hline Direct of NR & 0.005 & 0.066 & 0.119 & 0.015 & 0.118 & -0.056 & -0.056 \\
\hline Ind.through MTG & -0.047 & -0.070 & 0.094 & -0.023 & 0.113 & 0.007 & 0.007 \\
\hline Ind.through PH & -0.002 & -0.011 & 0.001 & 0.011 & -0.063 & -0.033 & -0.033 \\
\hline Ind.through FPIH & -0.015 & 0.011 & -0.003 & -0.018 & 0.000 & -0.004 & -0.004 \\
\hline Ind.through TNP & 0.180 & 0.261 & 0.196 & 0.189 & 0.320 & 0.204 & 0.204 \\
\hline Ind.through NOGP & 0.017 & 0.013 & 0.031 & 0.048 & -0.013 & -0.031 & -0.031 \\
\hline Ind.through NTWOGP & 0.144 & 0.092 & 0.143 & 0.122 & 0.061 & 0.165 & 0.165 \\
\hline Ind.through NTGP & 0.212 & 0.231 & 0.155 & 0.254 & 0.082 & 0.214 & 0.214 \\
\hline Total & 0.494 & 0.593 & 0.737 & 0.598 & 0.619 & 0.464 & 0.464 \\
\hline Direct of NOGP & 0.042 & 0.029 & 0.081 & 0.105 & 0.067 & -0.064 & -0.064 \\
\hline Ind. through MTG & -0.246 & -0.259 & -0.188 & -0.242 & -0.047 & -0.345 & -0.345 \\
\hline Ind.through PH & 0.002 & -0.005 & -0.002 & 0.003 & -0.059 & -0.021 & -0.021 \\
\hline Ind.through FPIH & -0.009 & 0.011 & -0.003 & -0.033 & -0.020 & -0.040 & -0.040 \\
\hline Ind.through TNP & 0.223 & 0.270 & 0.197 & 0.186 & 0.357 & 0.285 & 0.285 \\
\hline Ind.through NR & 0.002 & 0.029 & 0.046 & 0.007 & 0.023 & -0.027 & -0.027 \\
\hline Ind.through NTWOGP & 0.175 & 0.099 & 0.155 & 0.131 & 0.070 & 0.249 & 0.249 \\
\hline Ind.through NTGP & 0.154 & 0.103 & -0.002 & 0.122 & -0.021 & 0.187 & 0.187 \\
\hline Total & 0.343 & 0.277 & 0.285 & 0.279 & 0.237 & 0.222 & 0.222 \\
\hline Direct of NTWOGP & 0.244 & 0.145 & 0.242 & 0.195 & 0.114 & 0.314 & 0.314 \\
\hline Ind. through MTG & -0.295 & -0.299 & -0.193 & -0.255 & -0.071 & -0.388 & -0.388 \\
\hline Ind.through PH & 0.000 & -0.010 & -0.002 & -0.002 & -0.045 & -0.013 & -0.013 \\
\hline Ind.through FPIH & -0.018 & 0.015 & -0.005 & -0.049 & -0.034 & -0.073 & -0.073 \\
\hline Ind.through TNP & 0.273 & 0.349 & 0.268 & 0.236 & 0.529 & 0.341 & 0.341 \\
\hline Ind.through NR & 0.003 & 0.042 & 0.070 & 0.010 & 0.063 & -0.030 & -0.030 \\
\hline Ind.through NOGP & 0.030 & 0.020 & 0.052 & 0.070 & -0.041 & -0.051 & -0.051 \\
\hline Ind.through NTGP & 0.214 & 0.178 & 0.033 & 0.147 & 0.065 & 0.265 & 0.265 \\
\hline Total & 0.454 & 0.441 & 0.468 & 0.355 & 0.581 & 0.369 & 0.369 \\
\hline Direct of NTGP & 0.527 & 0.494 & 0.420 & 0.486 & 0.248 & 0.584 & 0.584 \\
\hline Ind.through MTG & -0.141 & -0.190 & 0.076 & -0.026 & -0.009 & -0.087 & -0.087 \\
\hline Ind.through PH & 0.002 & -0.008 & 0.001 & 0.026 & -0.018 & -0.076 & -0.076 \\
\hline Ind.through FPIH & -0.003 & 0.007 & 0.002 & -0.003 & -0.009 & -0.009 & -0.009 \\
\hline Ind.through TNP & 0.229 & 0.278 & 0.163 & 0.181 & 0.392 & 0.285 & 0.285 \\
\hline Ind.through NR & 0.002 & 0.031 & 0.044 & 0.008 & 0.039 & -0.021 & -0.021 \\
\hline Ind.through NOGP & 0.012 & 0.006 & 0.000 & 0.026 & 0.006 & -0.021 & -0.021 \\
\hline Ind. through NTWOGP & 0.099 & 0.052 & 0.019 & 0.059 & 0.030 & 0.143 & 0.143 \\
\hline Total & 0.731 & 0.674 & 0.727 & 0.761 & 0.681 & 0.803 & 0.803 \\
\hline Residual & 0.40453 & 0.43048 & 0.2884 & 0.45370 & 0.30415 & 0.38127 & 0.38127 \\
\hline K value & 0.00682 & 0.00682 & 0.0068 & 0.00682 & 0.00682 & 0.00682 & 0.00682 \\
\hline $\mathrm{R}^{2}$ & 0.83636 & 0.81469 & 0.9168 & 0.79415 & 0.90749 & 0.85463 & 0.85463 \\
\hline
\end{tabular}

According to Johnson and Wichern (1992), the positive factorial loads (higher or equal to 0.7 ) indicate environments with elevated correlation and similar among one another. Besides, the observed environmental indexes were positive for these three environments, followed by elevated communality; thereby, classifying the environments as favorable. The second factor for the environment Independência-RS showed factorial load of 0.938; however, there was no grouping with the other environments, suggesting that this environment is isolated and has negative environmental index and classification as unfavorable. Thus, the results for environmental classification as favorable and unfavorable, and similarity degree were consistent. Such results are satisfactory and important for coherent environmental indication. Study conducted by Mendonça et al. (2007), concluded that environmental stratification achieved through factor analysis is more selective than the traditional method of Lin. The results obtained through Annicchiarico's method and factor analysis for environment stratification were similar, once they revealed the same grouping pattern and similarity for the environments. Therefore, for both methods, the environments Tapera-RS, Derrubadas-RS and Frederico Westphalen-RS influenced similarly and positively on productive behavior of the tested genotypes, suggesting that the interaction effect was reduced to non-significant, evidencing no differences among environments. Thus, it is assumed that effects of primary and secondary grain yield components are also constant in these conditions. On the other hand, in Independência-RS, the genotypes presented specific behavior, due to the conditions they were subjected, being unfavorable for expressing the grain yield trait. Pre-supposition to explain 
and authenticate favorable and unfavorable environment conditions on yield components may be measured and corroborated based on phenotypic correlations.

\section{Pearson's linear correlation for joint analysis of environments (joint)}

The phenotypic correlations indicate the linear dependence between two traits, considering that genotypes may be modified physiologically and morphologically according to the conditions imposed by the growing environment (Mauad et al., 2010). The Pearson's linear correlation was affected for nine grain yield-related traits and revealed 36 associations, of each, 30 were significant in the joint analysis (method I).

The stratification between favorable and unfavorable environments (method II) revealed 31 significant associations for favorable environments, and 22 significant associations for unfavorable environments (Table 5). Thus, the environmental edaphoclimatic conditions influenced the phenotypic associations between these traits, evidencing that the favorable conditions for development of soybean plants contribute to increment of significant associations, when compared to joint analysis.

In the stratification of linear associations for growing environment (method III), the environment IndependênciaRS showed 22 significant linear associations. The environment Tapera-RS showed 19 significant correlations. In Derrubadas-RS, 22 significant associations were observed, and in the environment Frederico Westphalen-RS, 15 significant associations (Table 6). Based on the number of significant phenotypic associations, it is possible to highlight that, even in the environments grouped as favorable, the correlations between pairs of traits do not reveal the same linear tendency. Thus, even in regions where grain yield is stable in genotypes as function of the environment, modifications in the associations between traits are observed due to alterations in morphologic and physiologic response of plants. In addition, this result may be due to the higher pairs of covariances attributed to lower deviations (Cruz et al., 2012).

In the joint correlation analysis (method III), greater importance will be given for significant correlations between the pairs with grain yield, considering the trait nature and agronomic importance, and for the pairs of correlations that remained significant for the three evaluation methods.

\section{Pearson's linear correlation for individual growing environment}

The joint analysis of stratification in favorable and unfavorable environments and stratification for environment (Independência-RS, Tapera-RS, Derrubadas-RS and Frederico Westphalen-RS), the grain yield (GY) revealed positive associations with intermediate to strong magnitudes with total number of pods per plant (TNP), number of ramifications (NR), number of two-grain pods (NTWOGP), and number of three-grains pod (NTGP) (Table 5 and Table 6). Thus, there is a tendency that grain yield may be increased due to the raise in number of ramifications, since the increment in these structures favors the development of reproductive structures, where the pods are formed, and consequently increasing the number of pods per plant. Once these pods contain by two or three grains, number of grains per plant and consequently, grain yield will rise. This result corroborates with Perini et al. (2012) and Nogueira et al. (2012) which revealed that soybean plant with indeterminate growth habit exhibited a high correlation of grain yield with the total number of pods and grains per plant.

The modification of the correlations between the same characters due to the growing environments suggests that secection of soybean genotypes based on yield traits will be efficient if performed in the environment of adaptation of the genotype (Nogueira et al., 2012). Thus, grain yield revealed positive and intermediate associations with mass of thousand grains in the joint analysis (method I), stratification of favorable environments (method II), and in environments Tapera-RS and Frederico Westphalen-RS (method II). The low and impertinent occurrence of significant correlation between grain yield and mass of a thousand grains may be explained by the compensation promoted by soybean plants, increasing or reducing grains size in function of the number of pods, where they are developed (Taware et al., 1997; Carvalho et al., 2002).

The phenotypic correlation between grain yield and plant height was significant only for joint analysis, and for the environment Derrubadas-RS, revealing intermediate and positive magnitudes. As a result, this trait cannot be prioritized for selecting superior genotypes due to its instability to the environmental changes. In addition, the joint analysis was tendentious to reveal the actual phenotypic correlations between plant height and grain yield. Study conducted by Nogueira et al. (2012), demonstrated a significant change in the magnitude and direction of correlation between plant height at maturity and grain yield as a function of two sowing times.

The grain yield showed a positive and intermediate association with the number of one grain pods in the joint analysis (method I), and in the favorable environment (Method II). For the other forms of stratification, no significant differences were observed. A probable explanation for this modification is the edaphoclimatic factors in each environment, which results in genotype $x$ environment interaction. In addition, non-significant correlation coefficients between the characters do not imply a lack of correlation, but they express the absence of a linear relationship between them (Cruz et al., 2004).

In joint analysis, stratification of favorable and unfavorable environments (Independência-RS, Tapera-RS, DerrubadasRS, Frederico Westphalen-RS), the total number of pods per plant revealed positive associations of intermediate to very strong magnitudes with number of ramifications, number of one-grain pods, number of two-grain pods and number of three-grain pods (Table 5 and 6). Therefore, when the total number of pods per plant increased, there was a tendency to increase the number of pods with one- and two-grain, since the production of photoassimilates by the plant must meet the need imposed by the greatest number of drains. Similar results were found by Pipolo et al. (2005), which concluded that plants with higher number of pods with two grains showed higher number of pods per plant and, consequently, higher grain mass per plant.

The trait number of ramifications revealed intermediate to strong positive associations with number of two-grain pods and number of three-grain pods. In this way, the number 
and size of the ramifications provide an increase in the number of buds, forming reproductive structures, increasing the number of fertile nodes in the plant. The number of pods contributes to increase grain yield (Rambo et al., 2003). The results corroborate with Szareski et al. (2015), who evidenced strong and positive correlation between number of ramifications per plant and number of pods with two- and three-grain and grain yield.

The plant height presented positive and strong phenotypic correlation with first pod insertion height. Therefore, the elevation of plant height tends to increase the height of first pod insertion, since tall plants have a denser canopy and restrict the interception of photosynthetically active radiation in the lower parts of the plant. This causes the increase of the self-shadowing, resulting in senescence of leaves and abscission of reproductive structures, raising the height of the first viable legume (Casaroli et al., 2007). According to Miranda (1998), the soybean ideotype should present lower first pod insertion height and higher plant height, as it increases the number of nodes per plant and consequently the formation of reproductive structures.

In the same way for methods I, II and III, the trait number of one-grain pods showed a positive and strong correlation with number of two-grain pods. Similar results were observed by Carpentieri-Pípopo et al. (2005), where the positive associations between these characters occurred for 34 soybean strains. However, the increase in the number of one-grain pods is not desirable for grain yield. Therefore, preference is given to soybean genotypes with three-grain per pod (Navarro Junior and Costa, 2002).

The first pod insertion height revealed intermediate and negative association with number of two-grain pods. A similar result was obtained by Szareski et al. (2015), where strong and negative correlation between the first pod insertion height and the number of pods with two grains was observed. The interpretation of the magnitude of a phenotypic association may result in erroneous conclusion, since a high correlation between two characters may be due to the indirect effect of other characters, being necessary to deploy the linear tendencies in direct and indirect effects of each character, also considering the growing environments (Carvalho et al., 2002). Some characters are less influenced by environmental effects. They do not alter their behavior facing modifications imposed by the environment, indicating applicability for indirect selection. Thus, the study showed that the direct effects of mass of a thousand grains, total number of pods, number of two grains pods, and number of three grains pods remained constant and positive in both stratification methods (Table 7). Studies by lqbal et al. (2003), and Nogueira et al. (2012) observed that the greater direct phenotypic effects on grain yield were evidenced for number of pods per plant, mass of one hundred grains, and number of grains per pod. Therefore, these characters should be prioritized for selection of superior soybean genotypes due to the stability of response in different environmental conditions.

\section{Direct and indirect phenotypic effects for joint analysis of environments (joint), environmental stratification (FVEV and UFEV) and for environments}

It was observed that mass of thousand grains had indirect negative effects on the total number of pods per plant, number of two-grain pods and number of three-grain pods. On the other hand, for total number of pods per plant, there were negative indirect effects with the mass of a thousand grains, and positive with the number of two- and threegrain pods. The results corroborate with Santos et al. (1995) and Nogueira et al. (2012) which presented negative indirect effects through the total number of pods on the mass of thousand grains.

The number of two-grain pods presented positive indirect effects with total number of pods and number of threegrain pods, and indirect negative effect with the mass of a thousand grains. The number of pods with three-grains was influenced by indirect effects of the mass of a thousand grains and the total number of pods. Thus, it is believed that the total number of pods presented the most direct and indirect influence on grain yield. This result corroborates with Board et al. (1997), Mohammand et al. (2010) and Alcantara Neto et al. (2011), where they verified that the number of pods per plant is determinant for grain yield components, since it presents greater direct effect on yield and indirect effect on other primary and secondary yield components.

The direct effects of number of ramifications and number of one-grain pods on grain yield were positive in the joint analysis and in the favorable environments (Table 7). However, the direct effects of these traits are negative in the unfavorable environment (Method II). Regarding the indirect effects acting on these traits, it is observed that both were indirectly influenced by the total number of pods per plant, number of two- and three-grain pods. Thus, the sense alteration of direct effects of these traits due to edaphoclimatic conditions for genotypes development corroborates with the need to investigate these effects separately. Therefore, environmental stratification is efficient to infer the selection strategies more accurately, since environments classified as favorable maintained their constant effects.

The direct effects observed for the plant height and first pod insertion height on grain yield did not maintain steady pattern of behavior. Thus, in the stratification of favorable environments, the environments that included in this group revealed effects of different magnitude and direction (Table 7). Therefore, these traits suffer greater influence of genotypes $x$ environments interaction and the joint analysis does not indicate accurate inferences about their effects. On the other hand, the indirect effects that influenced these traits remained constant, being the mass of a thousand grains responsible for positive effects, and the total number of pods per plant with first pod insertion height. The indirect effects of other traits barely contributed to the main trait, and the best selection strategy should consist of traits with significant indirect effects (Cruz et al., 2004). With the results achieved in this study, it can be inferred that the applicability of path analysis based on environmental stratification may minimize the effects resulting from genotypes $x$ environments interactions on the cause and effect relations of soybean. Then, the number of analyzes may be reduced, keeping the precision and efficiency of the information. It allows the breeder to outline strategies and indirect selections that are more efficient and weighted by growing environment effects, increasing the probability of selecting soybean genotypes superior to the traits of agronomic interest. 


\section{Materials and methods}

\section{Plant materials}

The tests were conducted in the agricultural year of 2013/14 in four growing sites in Rio Grande do Sul, Brazil (Table 1). The environments used in the study satisfied the main soybean production areas of Rio Grande do Sul.

\section{Experimental design and experimental procedure}

The experimental arrangement adopted was randomized blocks, in factorial scheme (11 x 4), including eleven soybean genotypes in four environments, assigned in four repetitions. The environments and location of study were Independência-RS, Tapera-RS, Derrubadas-RS and Frederico Westphalen-RS. The genotypes used were: Nidera 5445 IPRO, CCGL 6029 IPRO, Don Mario 6458 IPRO, Brasmax 6160 IPRO, Coodetec 2611 IPRO, Nidera 6211 RR, CCGL S13/03 RR, Don Mario Apolo RR, Brasmax Alvo RR, Coodetec 2585 RR and TMG 7161 RR. All used genotypes are of undetermined growing habit and maturation group ranged from 5.3 to 6.3.

The plots were sown between November $15^{\text {th }}$ and $19^{\text {th }}$ of 2013. Sowing was manually done, after opening of grooves with the tractor-seeder assembly, with $300 \mathrm{~kg} \mathrm{ha}^{-1}$ of $\mathrm{N}$ $\mathrm{P}_{2} \mathrm{O}_{5}-\mathrm{K}_{2} \mathrm{O}$ in the formulation 02-25-25 as base fertilization. The criteria for density and sowing timing were established according to technical recommendation for each genotype, being the same criteria attended for sowing time and soy macro-region.

The experimental unity (EU) was constituted of five lines of three meters length, spaced 45 centimeters. Two lines were used as borders, and 0.5 meters of each experimental unity extremity were discarded to minimize border effects. The EU had useful area of 2.7 square meters. After physiologic maturation, 10 representative plants were randomly sampled and the yield components were measured.

\section{Traits measured}

The evaluated traits were: plot grain yield (GY): Measured from mass of grains per plant, pondered by number of plants in the plot useful area, with humidity corrected to $13 \%$, obtaining the mass of grains per plot, with results expressed in $\mathrm{kg} \mathrm{plot}^{-1}$; Mass of a thousand grains (MTG): Obtained through manual counting of eight repetitions of 100 grains, expanding for a thousand grains mass, and results expressed in grams (g); Plant height (PH): It was measured the length from soil surface and tip of the main stem, aided by a ruler, with results expressed in centimeters $(\mathrm{cm})$; First pod insertion height (FPIH): obtained through the length between soil surface and the insertion of the first pod, aided by a ruler, with results expressed in centimeters $(\mathrm{cm})$; Total number of pods per plant (TNP): obtained through counting the total number of pods per plants, with results expressed in unities. Number of ramifications or branches (NR): it was counted the number of ramifications bigger than 10 centimeters, with results expressed in unities. Number of one-grain pods (NOGP): Obtained through counting the total number of pods with one grain, results in unities; Number of two-grain pods (NTWOGP): Obtained through counting the total number of pods with two grains, results in unities; Number of three-grain pods (NTGP): Obtained through counting the total number of pods with three grains, results in unities.

\section{Statistical analysis}

The data was subjected to the variance analysis through the F-test with $p \leq 0.05$ of probability. Previously the variance analysis, pre-suppositions of the statistic model was individually verified for each variable. This way, the method I considered the joint analysis of all environments, method II, the environmental stratification (favorable and unfavorable environment), and method III, the stratification for environment (Independência-RS, Tapera-RS, Derrubadas-RS and Frederico Westphalen-RS). In the presence of significant interaction between genotypes and growing environments for the plot grain yield, the environmental stratification was made through the methods of Annicchiarico (1992) and analysis of factors (Johnson and Wichern, 1992; Murakami and Cruz, 2004).

The Pearson's liner correlation coefficients were also estimated and the coefficient magnitudes were classified according to Carvalho et al. (2004). Values equal to 0.00 correspond to null correlation; correlation from 0.00 to 0.30 : weak; correlations from 0.30 to 0.60 : intermediate; correlation from 0.60 to 0.90 : strong; correlation from 0.90 to 1 : very strong. Intending to deploy linear coefficients in cause and effect associations, path analysis was performed. In preceding path analysis, it was affected the multicollinearity diagnostic of the matrices of correlations based on their number of conditions (NC) correspond to the ratio between bigger and lower matrix eigenvalue according to Cruz et al. (2012). Thus, results for both tested matrices revealed collinearity of moderate to strong, considering that the number of conditions showed an interval from 100 to 1000; therefore, classifying the multicollinearity as moderated to strong (Montgomery and Peck, 1981). As diagnostic for presence of multicollinearity, the $k$ constant was introduced to the $X^{\prime} X$ matrix diagonal, which consists in a small quantity added to the diagonal elements of the $X^{\prime} X$ matrix. The $k$ value used was established as the smallest value capable of stabilize most path analysis estimators (Carvalho et al., 1999).

For path analysis estimates, grain yield per plot was considered as dependent trait and the other traits such as mass of a thousand grains, plant height, first pod insertion height, total number of pods, number of ramifications, number of one grain pods, number of two grains pod, number of three grains pods were considered as primary variables or explainable. Thus, the deployment of correlation between the dependent trait and the explainable were affected, according to Cruz et al. (2004). The analyses were realized through the software Genes (Cruz, 2013).

\section{Conclusion}

The growing environments Tapera-RS, Derrubadas-RS and Frederico Westphalen-RS were classified as favorable for soybean cultivation. The traits total number of pods per plant, number of ramifications, number of two- and threegrain pods showed significant linear correlations with grain yield in both methods of analysis. The stratification applied for path analysis and groups of favorable and unfavorable environments was efficient to estimate accurate direct and 
indirect effects for soybean grain yield. The mass of thousand grains and plant height associated with grain yield were more influenced by growing environmental effects. Total number of pods should be prioritized for selecting superior soybean genotypes due to its direct and indirect effects on grain yield, as it was constant in both environmental stratification methods. Direct effects of number of ramifications and number of one-grain pods on grain yield present distinct senses in favorable and unfavorable environments.

\section{Acknowledgements}

The first author acknowledges the fellowship funded by Federal University of Santa Mariacampus Frederico Westphalen (UFSM) Brazil, and Breeding Laboratory and Plant Production (LMGPP).

\section{References}

Alcantara Neto F, Gravina GA, Monteiro MMS, Morais FB, Petter FA, Albuquerque JAA (2011) Path analysis of soybean yield in the micro region of Alto Médio Gurguéia. Comunicata Scientiae. 2(2): 107-112.

Annicchiarico P (1992) Cultivar adaptation and recommendation from alfalfa trials in Northern Italy. Journal of Genetics and Breeding. 46: 269-278.

Board JE, Kang MS, Harville BG (1997) Path analysis identify indirect selection criteria for yield of late planted soybean. Crop Science. 37(3): 879-884.

Carneiro PCS (1998) Novas metodologias de análise de adaptabilidade e estabilidade de comportamento.Thesis (Doctorate) Federal Universityof Viçosa, Viçosa,168p.

Carpentieri-Pípolo V, Gastaldi LF, Pipolo AE (2005) Phenotypic correlations between quantitative characteristics in soybean. Semina: Ciências Agrárias. 26(1): 11-16.

Carvalho ADF, Silva GO, Perreira RB, Pinheiro JB (2014) Analysis of factors and bissegmented regression in the study of adaptability and stability in carrots. Revista Ceres. 61(6): 932-940.

Carvalho CGP, Arias CAA, Toledo JFF, Oliveira MF, Vello NA (2002) Correlation and path analyses in soybean lines sowed at different sowing dates. Brazilian Journal of Agricultural Research. 37(3): 311-320.

Carvalho CGP, Oliveira VR, Cruz CD, Casali VWD (1999) Path analysis under multicollinearity in green pepper.Brazilian Journal of Agricultural Research34(4): 603-613.

Carvalho FIF, Lorencerti C, Benin G (2004) Estimativas e Implicações da Correlação. Pelotas. Ed. Universitária da UFPEL, 142p.

Carvalho IC, Souza VQ,Nardino M, Follmann DN, Silva ADB, Szareski VJ, Pelegrin AJ, Ferrari M, Olivoto T (2015) Associations phenotypic between physiological traits of soybean contrasting growth habits. Global Science and Technology. 08(3): 30-40.

Casaroli D, Fagan EB, Simon J, Medeiros SP, Manfron PA, Douradoneto D, Van Lier QJ, Muller L, Martin TN (2007) Solar radiation and physiologics aspects in soybean - a review. Revista da FZVA, Uruguaiana. 14:1 02-120.

Cruz CD (2013) GENES - a software package for analysis in experimental statistics and quantitative genetics. Acta Scientiarum, Maringá. 35(3): 271-276.
Cruz CD, Regazzi AJ, Carneiro PCS (2012) Modelos biométricos aplicados ao melhoramento genético. UFV, Viçosa, Brasil, 514p.

Cruz CD, Regazzi AJ, Carneiro PCS (2004) Modelos biométricos aplicados ao melhoramento genético, Ed 3 . Viçosa, $480 \mathrm{p}$.

Falconer DS(1981)Introdução à genética quantitativa. Viçosa: UFV, 279p.

Iqbal S, Mahmood T, Muhammad TA, Muhammad A, Muhammad S (2003) Path coefficientanalysis in different genotypes of soybean (Glycine max (L.) Merrill). Pakistan Journal of Biological Science. 12: 1085-1087.

Johnson RA, Wichern DW (1992) Applied multivariate statistical analysis. New Jersey: Prentice Hall Inc., 642p.

Maluf JRT (2000) A new climatic classification for the State of Rio Grande do Sul, Brazil. Brazilian Journal of Agrometeorology. 8(1): 141-150.

Mauad M, Silva TLB, Neto AIA, Abreu VG (2010) Influence of seeding rate on agronomic traits of soybean. Revista Agrarian. 3(9): 175-181.

Mendonça O, Carpentieri-Pípolo V, Garbuglio DD, Fonseca Junior NS (2007) Factor analysis and environmental stratification in the assessment of soybean adaptability and stability. Brazilian Journal of Agricultural Research. 42:1567-1575.

Miranda GV (1998) Diversidade genética e desempenho de cultivares de soja como progenitores. Thesis (Doctorate). Federal Universityof Viçosa, Viçosa. 117p.

Mohammad S,Bahman A(2010)Sequential path model for grain yield in soybean.NotulaeScientiaBilogicae2(3): 101109.

Montgomery DC, Peck EA, Vining GG (1981) Introduction to linear regression analysis New York: John Wiley \& Sons, 504p.

Murakami DM, Cruz CD (2004) Proposal of methodologies for environment stratification and analysis of genotype adaptability. Crop Breeding and Applied Biotechnology. 4(1): 7-11.

Murakami DM, Cardoso AA, Cruz CD, Bizão N( 2004)Some considerations about two methodologies for stability and adaptability analysis. Ciência Rural. 34(1): 71-78.

Navarro Júnior HM, Costa JA (2002) Relative contribution of yield components for grain production in soybean. Brazilian Journal of Agricultural Research. 37(3): 269-274.

Nogueira APO, Sediyama T, Souza LB, Hamawaki OT, Cruz CD, Pereira DG, Matsuo E (2012) Path analysis and correlations among traits in soybean grown in two dates sowing. Bioscience Journal. 28(6): 877-888.

Perini LJ, Fonseca Jr NS, Destro D, Prete CEC (2012) Components of the production in soybean cultivars with determinate and indeterminate growth. Semina: Ciências Agrárias. 33(1): 2531-2544.

Pipolo VC, Gastaldi LF, Pipolo AE (2005) Phenotypic correlations between quantitative characteristics in soybean. Semina: Ciências Agrárias. 26(1): 11-16.

Ramalho MAP, Santos JB, Zimmermann MJO (1993) Genética quantitativa em plantas autógamas: aplicações ao melhoramento do feijoeiro. Goiânia: UFG. 271 p.

Ramalho MAP, Abreu AFB, Santos JB, Nunes JAR (2012) Aplicações da genética quantitativa no melhoramento de espécies autógamas. 1a ed. Lavras: Editora UFLA, 522p. 
Rambo L, Costa JA, Pires JLF,Parcianello G, Ferreira FG (2003) Soy bean yield response to plant arrangement. Ciência Rural 33(3): 405-411.

Santos CAF, Reis MS, Cruz CD, Sediyama CS,Sediyama T (1995) Adequação de modelos noestudo de coeficiente de trilha nos componentes primários e secundário de progênies F6 de soja (Glycinemax (L.) Merril). Revista Ceres 42: 111-121.

Santos HG, Jacomine PKT, Anjos LHC, Oliveira VA, Oliveira JB, Coelho MR, Lumbreras JF, Cunha TJF (2006) Sistema brasileiro de classificação de solos. Rio de Janeiro: Embrapa Solos. 306 p.

Schmildt ER, Cruz CD (2005) Adaptability and stability of maize using Eberhart/ Russell and Annicchiarico methods.R evista Ceres. 52(299): 45-58.
Shukla S, Pushpendra SK (1998) Correlation and path coefficient analysis of yield and its components in soybean (Glycine max L. Merrill.). Soybean Genetics Newsletter. 25: 67-70.

Silva WCJE, Duarte JB (2006) Statistical methods to study phenotypic adaptability and stability in soybean. Brazilian Journal of Agricultural Research. 41(1): 23-30.

Szareski VJ, Souza VQ, Carvalho IR, Nardino M, Follmann DN, Demari GH, Ferrari M, Olivoto T (2015) Growing environment and its effects on morphological characters and dietetic soy. Revista Brasileira de Agropecuária Sustentável. 5(2): 79-88.

Taware SP, Halvankar GB, Raut VM, Patil VP (1997) Variability, correlation and path analysis in soybean hybrids. Soybean Genetics Newsletter. 24:96-98.

Wright S (1921) Correlation and causation. Journal of Agricultural Research. 20: 557-585. 